\title{
Liouville theorem for elliptic equations with mixed boundary value conditions and finite Morse indices
}

\section{Xueqiao Wang and Xiongjun Zheng*}

*Correspondence: xjzh1985@126.com

Department of Mathematics, Jiangxi Normal University, Nanchang,

Jiangxi 330022, P.R. China

\begin{abstract}
In this paper, we establish Liouville type theorem for boundedness solutions with finite Morse index of the following mixed boundary value problems: $-\Delta u=|u|^{p-1} u$ in $\mathbb{R}_{+,}^{N} \frac{\partial u}{\partial v}=|u|^{q-1} u$ on $\Gamma_{1}, \frac{\partial u}{\partial v}=0$ on $\Gamma_{0}$, and $-\Delta u=|u|^{p-1} u$ in $\mathbb{R}_{+,}^{N} \frac{\partial u}{\partial v}=|u|^{q-1} u$ on $\Gamma_{1}$, $u=0$ on $\Gamma_{0}$, where $\mathbb{R}_{+}^{N}=\left\{x \in \mathbb{R}^{N}: x_{N}>0\right\}, \Gamma_{1}=\left\{x \in \mathbb{R}^{N}: x_{N}=0, x_{1}<0\right\}$ and $\Gamma_{0}=\left\{x \in \mathbb{R}^{N}: x_{N}=0, x_{1}>0\right\}$. The exponents $p, q$ satisfy the conditions in Theorem 1.1.
\end{abstract}

Keywords: Sobolev-Hardy inequality; minimizer; radial symmetry; decaying law

\section{Introduction}

In this paper, we establish a Liouville type theorem in the upper half space $\mathbb{R}_{+}^{N}$ with mixed boundary conditions.

In a seminal paper [1], Gidas and Spruck considered positive solutions of the nonlinear elliptic problem

$$
-\Delta u=|u|^{p-1} u, \quad \text { in } \mathbb{R}^{N}
$$

for the subcritical case: $1<p<\frac{N+2}{N-2}, N \geq 3$. Although when $p=\frac{N+2}{N-2}$, the critical case, problem (1.1) possesses a family of solutions, it was proved in [1] that problem (1.1) has no positive solutions. This is the so-called Liouville type theorem. Soon afterward, similar results were established in [2] for positive solutions of the subcritical problem in the upper half space $\mathbb{R}_{+}^{N}$ :

$$
\left\{\begin{array}{ll}
-\Delta u=|u|^{p-1} u & \text { in } \mathbb{R}_{+}^{N}, \\
u=0 & \text { on } \partial \mathbb{R}_{+}^{N}
\end{array} .\right.
$$

As soon as such results appeared, they received wide attention as regards the theory itself and its applications. Particularly, one may use it to establish a prior bound of solutions for nonlinear elliptic equations by the blow-up method, then various methods, such as topological degree, fix point theorems etc., can be used to obtain the existence of solutions for such problems; see for instance [1] and [2].

On the other hand, it is puzzling if problems (1.1) and (1.2) admit sign-changing solutions. A partial answer came from [3] by assuming additionally that solutions have finite

(c) 2015 Wang and Zheng. This article is distributed under the terms of the Creative Commons Attribution 4.0 International License (http://creativecommons.org/licenses/by/4.0/), which permits unrestricted use, distribution, and reproduction in any medium, provided you give appropriate credit to the original author(s) and the source, provide a link to the Creative Commons license, and indicate if changes were made. 
Morse indices. It was proved in [3] that problems (1.1) and (1.2) do not possess nontrivial bounded solution with finite Morse index provided $1<p<\frac{N+2}{N-2}$. In applications, fortunately, one may find critical points with finite Morse indices by the mountain pass theorem and saddle point theorem and so on, it allows one to establish for instance as in [4], the existence result for indefinite nonlinearities.

Recently, it is investigated by many authors various type of Liouville theorems for solutions with finite Morse indices, such as problems with Neumann boundary condition, Dirichlet-Neumann mixed boundary and nonlinear boundary conditions etc.; see [5-10] and references therein.

Inspired by previous work, we study in this paper Liouville type results for the following nonlinear Neumann mixed boundary value problem:

$$
\begin{cases}-\Delta u=|u|^{p-1} u & \text { in } \mathbb{R}_{+}^{N} \\ \frac{\partial u}{\partial v}=|u|^{q-1} u & \text { on } \Gamma_{1} \\ \frac{\partial u}{\partial v}=0 & \text { on } \Gamma_{0}\end{cases}
$$

as well as the nonlinear Dirichlet mixed boundary value problem

$$
\begin{cases}-\Delta u=|u|^{p-1} u & \text { in } \mathbb{R}_{+}^{N}, \\ \frac{\partial u}{\partial v}=|u|^{q-1} u & \text { on } \Gamma_{1} \\ u=0 & \text { on } \Gamma_{0}\end{cases}
$$

where $\mathbb{R}_{+}^{N}=\left\{x \in \mathbb{R}^{N}: x_{N}>0\right\}, \Gamma_{1}=\left\{x \in \mathbb{R}^{N}: x_{N}=0, x_{1}<0\right\}$ and $\Gamma_{0}=\left\{x \in \mathbb{R}^{N}: x_{N}=0, x_{1}>\right.$ $0\}$.

It was proved in [11] that problems (1.3) and (1.4) have no positive solutions. In this paper, we deal with solutions of problems (1.3) and (1.4) with finite Morse indices, which are possibly sign-changing. For this purpose, we first define the Morse indices of solutions to problems (1.3) and (1.4), respectively. For problem (1.3), the Morse index of a solution $u$ is defined by

$$
\operatorname{dim}\left\{\varphi:\left\langle I^{\prime \prime}(u) \varphi, \varphi\right\rangle<0, \varphi \in C_{0}^{\infty}\left(\overline{\mathbb{R}_{+}^{N}}\right)\right\}
$$

where

$$
\left\langle I^{\prime \prime}(u) \varphi, \varphi\right\rangle=\int_{\mathbb{R}_{+}^{N}}|\nabla \varphi|^{2} d x-p \int_{\mathbb{R}_{+}^{N}}|u|^{p-1} \varphi^{2} d x-q \int_{\Gamma_{1}}|u|^{q-1} \varphi^{2} d x^{\prime} .
$$

For a solution $u$ of problem (1.4), we define its Morse index by

$$
\operatorname{dim}\left\{\varphi:\left\langle I^{\prime \prime}(u) \varphi, \varphi\right\rangle<0, \varphi \in C_{0}^{\infty}\left(\mathbb{R}_{+}^{N} \cup \Gamma_{1}\right)\right\}
$$

with $\left\langle I^{\prime \prime}(u) \varphi, \varphi\right\rangle$ defined in (1.5).

Our main results are as follows.

Theorem 1.1 If $1<p \leq \frac{N+2}{N-2}, 1<q \leq \frac{N}{N-2}$, and $(p, q) \neq\left(\frac{N+2}{N-2}, \frac{N}{N-2}\right)$, then problems (1.3) and (1.4) do not possess nontrivial bounded solution with finite Morse index. 
Theorem 1.1 will be proved in the next section. We first prove that a finite Morse index implies certain integrable conditions on $u$. Then by the Pohozaev identity, we show the nonexistence result.

\section{Proof of Theorem 1.1}

In this section, we establish the Liouville type theorem for bounded solutions of problems (1.3) and (1.4) with finite Morse indices, that is, we show that such solutions must be trivial. We assume in this section that $p, q$ in (1.3) and (1.4) satisfying $1<p \leq \frac{N+2}{N-2}, 1<q \leq \frac{N}{N-2}$, and $(p, q) \neq\left(\frac{N+2}{N-2}, \frac{N}{N-2}\right)$.

For $s>2 r>0$, we define a cut-off function $\phi_{r, s}$ as

$$
\phi_{r, s}= \begin{cases}0 & \text { for }|x|<r \text { or }|x|>2 s \\ 1 & \text { for } 2 r \leq|x| \leq s\end{cases}
$$

Moreover, we require $\left|\nabla \phi_{r, s}\right| \leq \frac{2}{r}$ for $r<|x|<2 r$ and $\left|\nabla \phi_{r, s}\right| \leq \frac{2}{s}$ for $s<|x|<2 s$. Then we have the following result; see also [4].

Lemma 2.1 Let $u$ be a solution of (1.3) or (1.4) with finite Morse index. Then there exists $R_{0}>0$ such that

$$
\left\langle I^{\prime \prime}(u) u \phi_{R_{0}, R}, u \phi_{R_{0}, R}\right\rangle \geq 0
$$

for any $R>2 R_{0}$.

Proof We suppose that the Morse index of $u$ is $k$. Now, we prove the conclusion by contradiction. Suppose on the contrary that inequality (2.1) does not hold, there would exist $r_{1}>0$ and $R_{1}>2 r_{1}$, such that

$$
\left\langle I^{\prime \prime}(u) u \phi_{r_{1}, R_{1}}, u \phi_{r_{1}, R_{1}}\right\rangle<0 .
$$

Similarly, we can find $r_{2}>2 R_{1}$ and $R_{2}>2 r_{2}$, such that

$$
\left\langle I^{\prime \prime}(u) u \phi_{r_{2}, R_{2}}, u \phi_{r_{2}, R_{2}}\right\rangle<0 .
$$

Iterating this procedure, we see that there exist $r_{k+1}>2 R_{k}$ and $R_{k+1}>2 r_{k+1}$, such that

$$
\left\langle I^{\prime \prime}(u) u \phi_{r_{k+1}, R_{k+1}}, u \phi_{r_{k+1}, R_{k+1}}\right\rangle<0 .
$$

By the above inequalities, we deduce that $u \phi_{r_{i}, R_{i}} \not \equiv 0$ for every $1 \leq i \leq k+1$. Moreover, since $\left\{u \phi_{r_{i}, R_{i}}\right\}_{i=1}^{k+1}$ have disjoint supports, we deduce that $\left\{u \phi_{r_{i}, R_{i}}\right\}_{i=1}^{k+1}$ are linearly independent. So the dimension of the linear space

$$
M=\operatorname{span}\left\{u \phi_{r_{i}, R_{i}}\right\}_{i=1}^{k+1}
$$

is $k+1$. We conclude from

$$
\left\langle I^{\prime \prime}(u) u \phi_{r_{i}, R_{i}}, u \phi_{r_{i}, R_{i}}\right\rangle<0
$$


for $1 \leq i \leq k+1$ that

$$
\left\langle I^{\prime \prime}(u) \varphi, \varphi\right\rangle<0
$$

for any $\varphi \in M$. This implies that the Morse index of $u$ is at least $k+1$, which contradicts the fact that the Morse index of $u$ is equal to $k$. The assertion follows.

Next, we show that a finite Morse index implies $u$ satisfying a certain integrable condition. More precisely, we have the following lemma.

Lemma 2.2 Assume that $1<p \leq \frac{N+2}{N-2}, 1<q \leq \frac{N}{N-2}$, and $(p, q) \neq\left(\frac{N+2}{N-2}, \frac{N}{N-2}\right)$. If $u$ is a bounded solution of problem (1.3) or (1.4) with finite Morse index, then we have

$$
\int_{\mathbb{R}_{+}^{N}}|u|^{p+1} d x<\infty, \quad \int_{\Gamma_{1}}|u|^{q+1} d x^{\prime}<\infty, \quad \int_{\mathbb{R}_{+}^{N}}|\nabla u|^{2} d x<\infty .
$$

Proof We only prove the results for problem (1.3). For problem (1.4), the proof can proceed similarly.

First, we prove that $\int_{\mathbb{R}_{+}^{N}}|u|^{p+1} d x<\infty$. By Lemma 2.1, there exists $R_{0}>0$, such that

$$
\left\langle I^{\prime \prime}(u) u \phi_{R_{0}, R}, u \phi_{R_{0}, R}\right\rangle \geq 0,
$$

for any $R>2 R_{0}$. That is,

$$
p \int_{\mathbb{R}_{+}^{N}}|u|^{p+1} \phi_{R_{0}, R}^{2} d x+q \int_{\Gamma_{1}}|u|^{q+1} \phi_{R_{0}, R}^{2} d x^{\prime} \leq \int_{\mathbb{R}_{+}^{N}}\left|\nabla\left(u \phi_{R_{0}, R}\right)\right|^{2} d x .
$$

Apparently,

$$
\int_{\mathbb{R}_{+}^{N}}\left|\nabla\left(u \phi_{R_{0}, R}\right)\right|^{2} d x=\int_{\mathbb{R}_{+}^{N}}|\nabla u|^{2} \phi_{R_{0}, R}^{2}+\left|\nabla \phi_{R_{0}, R}\right| u^{2}+2 u \phi_{R_{0}, R} \nabla u \nabla \phi_{R_{0}, R} d x .
$$

Multiplying (1.3) by $u \phi_{R_{0}, R}^{2}$ and integrating by parts, we obtain

$$
\int_{\mathbb{R}_{+}^{N}}|\nabla u|^{2} \phi_{R_{0}, R}^{2}+2 u \phi_{R_{0}, R} \nabla u \nabla \phi_{R_{0}, R} d x=\int_{\mathbb{R}_{+}^{N}}|u|^{p+1} \phi_{R_{0}, R}^{2} d x+\int_{\Gamma_{1}}|u|^{q+1} \phi_{R_{0}, R}^{2} d x^{\prime}
$$

By (2.2) and (2.3), we have

$$
\begin{aligned}
& (p-1) \int_{\mathbb{R}_{+}^{N}}|u|^{p+1} \phi_{R_{0}, R}^{2} d x+(q-1) \int_{\Gamma_{1}}|u|^{q+1} \phi_{R_{0}, R}^{2} d x^{\prime} \\
& \leq \int_{\mathbb{R}_{+}^{N}} u^{2}\left|\nabla \phi_{R_{0}, R}\right|^{2} d x \\
& \leq C_{0}+\frac{C}{R^{2}} \int_{\{R \leq|x| \leq 2 R\} \cap \mathbb{R}_{+}^{N}} u^{2} d x .
\end{aligned}
$$

In particular, we have

$$
(p-1) \int_{\mathbb{R}_{+}^{N}}|u|^{p+1} \phi_{R_{0}, R}^{2} d x \leq C_{0}+\frac{C}{R^{2}} \int_{\{R \leq|x| \leq 2 R\} \cap \mathbb{R}_{+}^{N}} u^{2} d x .
$$


If $N=2$, since $u$ is a bounded solution, the right hand side of (2.5) is bounded by a positive constant independent of $R$. So we have $\int_{\mathbb{R}_{+}^{N}}|u|^{p+1} d x<\infty$ by letting $R \rightarrow \infty$ in (2.5). Now for the case $N \geq 3$, we deduce from the Hölder inequality that

$$
\begin{aligned}
(p-1) \int_{\mathbb{R}_{+}^{N}}|u|^{p+1} \phi_{R_{0}, R}^{2} d x & \leq C_{0}+\frac{C}{R^{2}} \int_{\{R \leq|x| \leq 2 R\} \cap \mathbb{R}_{+}^{N}} u^{2} d x \\
& \leq C_{0}+C\left(\int_{\{R \leq|x| \leq 2 R\} \cap \mathbb{R}_{+}^{N}}|u|^{p+1} d x\right)^{\frac{2}{p+1}} \cdot R^{N \frac{p-1}{p+1}-2} .
\end{aligned}
$$

Suppose that $\int_{\mathbb{R}_{+}^{N}}|u|^{p+1} d x$ is infinite, then we deduce that

$$
\int_{B_{R}^{+}}|u|^{p+1} d x \leq C\left(\int_{B_{2 R}^{+}}|u|^{p+1} d x\right)^{\frac{2}{p+1}} \cdot R^{N \frac{p-1}{p+1}-2} .
$$

Let $\alpha=N \frac{p-1}{p+1}-2, \beta=\frac{2}{p+1}$, and $J(R)=\int_{B_{R}^{+}}|u|^{p+1} d x$. Iterating (2.7), we obtain

$$
J(R) \leq C R^{\alpha \gamma} J\left(2^{k+1} R\right)^{\beta^{k+1}}
$$

where $\gamma=1+\beta+\beta^{2}+\cdots+\beta^{k}$. The boundedness of $u$ implies that the right hand side of (2.8) is of order $R^{M}$ with

$$
M=\alpha \frac{1-\beta^{k+1}}{1-\beta}+N \beta^{k+1} \rightarrow \frac{\alpha}{1-\beta}
$$

as $k \rightarrow \infty$. Hence, we can choose $k$ large enough, such that $M<0$. Then it follows from (2.8) that

$$
J(R) \rightarrow 0
$$

as $R \rightarrow \infty$, which is impossible. So we get $\int_{\mathbb{R}_{+}^{N}}|u|^{p+1} d x<\infty$.

Next, we prove that $\int_{\partial \mathbb{R}_{+}^{N}}|u|^{q+1} d x^{\prime}<\infty$. In fact, we deduce from (2.4) that

$$
(q-1) \int_{\Gamma_{1}}|u|^{q+1} \phi_{R_{0}, R}^{2} d x^{\prime} \leq C_{0}+\frac{C}{R^{2}} \int_{\{R \leq|x| \leq 2 R\} \cap \mathbb{R}_{+}^{N}} u^{2} d x .
$$

If $N=2$, the right hand side of (2.9) is bounded by a positive constant independent of $R$. So we conclude that

$$
\int_{\Gamma_{1}}|u|^{q+1} d x^{\prime}<\infty
$$

by letting $R \rightarrow \infty$ in (2.9). Now for $N \geq 3$, we infer from (2.9) and the Hölder inequality that

$$
\begin{aligned}
& (q-1) \int_{\Gamma_{1}}|u|^{q+1} \phi_{R_{0}, R}^{2} d x^{\prime} \\
& \quad \leq C_{0}+\frac{C}{R^{2}} \int_{\{R \leq|x| \leq 2 R\} \cap \mathbb{R}_{+}^{N}} u^{2} d x
\end{aligned}
$$




$$
\begin{aligned}
& \leq C_{0}+C\left(\int_{\mathbb{R}_{+}^{N}}|u|^{p+1} d x\right)^{\frac{2}{p+1}} R^{N \frac{p-1}{p+1}-2} \\
& <\infty
\end{aligned}
$$

since $\int_{\mathbb{R}_{+}^{N}}|u|^{p+1} d x<\infty$ and $p \leq \frac{N+2}{N-2}$.

Finally, if we multiply (1.3) by $u$ and integrate on $\mathbb{R}_{+}^{N}$, then we get

$$
\int_{\mathbb{R}_{+}^{N}}|\nabla u|^{2} d x<\infty
$$

This completes the proof of this lemma.

The next lemma is the well-known local Pohozaev identity for elliptic problems with nonlinear boundary value condition.

Lemma 2.3 Let $u$ be a solution of (1.3) or (1.4). Then the following identity holds:

$$
\begin{aligned}
& \frac{N-2}{2} \int_{B_{R}^{+}}|\nabla u|^{2} d x-\frac{N}{p+1} \int_{B_{R}^{+}}|u|^{p+1} d x-\frac{N-1}{q+1} \int_{\Gamma_{1}^{R}}|u|^{q+1} d x^{\prime} \\
& =\frac{R}{2} \int_{\partial B_{R}^{+}}|\nabla u|^{2} d S-R \int_{\partial B_{R}^{+}}\left|\frac{\partial u}{\partial v}\right|^{2} d S-\frac{R}{p+1} \int_{\partial B_{R}^{+}}|u|^{p+1} d S \\
& \quad-\frac{R}{q+1} \int_{\partial \Gamma_{1}^{R}}|u|^{q+1} d S,
\end{aligned}
$$

where $B_{R}^{+}=\left\{x:|x|<R\right.$ and $\left.x_{N}>0\right\}, \partial B_{R}^{+}=\left\{x:|x|=R\right.$ and $\left.x_{N}>0\right\}, \Gamma_{0}^{R}=\left\{x \in \Gamma_{0}:|x|<R\right\}$, $\Gamma_{1}^{R}=\left\{x \in \Gamma_{1}:|x|<R\right\}, \partial \Gamma_{0}^{R}=\left\{x \in \Gamma_{0}:|x|=R\right\}, \partial \Gamma_{1}^{R}=\left\{x \in \Gamma_{1}:|x|=R\right\}$.

Proof The proof of this lemma is standard, we give it here for completeness. We deal only with problem (1.3). The proof for problem (1.4) is almost the same except that different boundary value conditions were used. We omit the details.

Multiplying (1.3) by $\langle x, \nabla u\rangle$ and integrating in $B_{R}^{+}$, we obtain

$$
-\int_{B_{R}^{+}} \Delta u\langle x, \nabla u\rangle=\int_{B_{R}^{+}}|u|^{p-1} u\langle x, \nabla u\rangle .
$$

A direct calculation shows that

$$
\begin{aligned}
& -\int_{B_{R}^{+}} \Delta u\langle x, \nabla u\rangle \\
& =\int_{B_{R}^{+}} \nabla u \nabla\langle x, \nabla u\rangle d x-\int_{\partial B_{R}^{+}} \frac{\partial u}{\partial v}\langle x, \nabla u\rangle d S-\int_{B_{R}^{0}} \frac{\partial u}{\partial v}\langle x, \nabla u\rangle d x^{\prime} \\
& =\int_{B_{R}^{+}}|\nabla u|^{2} d x+\frac{1}{2} \int_{B_{R}^{+}}\left\langle x, \nabla\left(|\nabla u|^{2}\right)\right\rangle d x-\int_{\partial B_{R}^{+}} \frac{\partial u}{\partial v}\langle x, \nabla u\rangle d S \\
& \quad-\frac{1}{q+1} \int_{\Gamma_{1}^{R}}\left\langle x^{\prime}, \nabla_{x^{\prime}}|u|^{q+1}\right\rangle d x^{\prime} \\
& =-\frac{N-2}{2} \int_{B_{R}^{+}}|\nabla u|^{2} d x+\frac{1}{2} \int_{\partial B_{R}^{+}}\langle x, v\rangle|\nabla u|^{2} d S-\int_{\partial B_{R}^{+}} \frac{\partial u}{\partial v}\langle x, \nabla u\rangle d S
\end{aligned}
$$




$$
\begin{aligned}
& +\frac{N-1}{q+1} \int_{\Gamma_{1}^{R}}|u|^{q+1} d x^{\prime}-\frac{1}{q+1} \int_{\partial \Gamma_{1}^{R}}\left|x^{\prime}, v\right||u|^{q+1} d S \\
= & -\frac{N-2}{2} \int_{B_{R}^{+}}|\nabla u|^{2} d x+\frac{R}{2} \int_{\partial B_{R}^{+}}|\nabla u|^{2} d S-R \int_{\partial B_{R}^{+}}\left|\frac{\partial u}{\partial v}\right|^{2} d S \\
& +\frac{N-1}{q+1} \int_{\Gamma_{1}^{R}}|u|^{q+1} d x^{\prime}-\frac{R}{q+1} \int_{\partial \Gamma_{1}^{R}}|u|^{q+1} d S .
\end{aligned}
$$

Next, we deduce

$$
\begin{aligned}
\int_{B_{R}^{+}} & |u|^{p-1} u\langle x, \nabla u\rangle d x \\
= & \frac{1}{p+1} \int_{B_{R}^{+}}\left\langle x, \nabla|u|^{p+1}\right\rangle d x \\
= & -\frac{N}{p+1} \int_{B_{R}^{+}}|u|^{p+1} d x+\frac{1}{p+1} \int_{\partial B_{R}^{+}}\langle x, v\rangle|u|^{p+1} d S \\
= & -\frac{N}{p+1} \int_{B_{R}^{+}}|u|^{p+1} d x+\frac{R}{p+1} \int_{\partial B_{R}^{+}}|u|^{p+1} d S .
\end{aligned}
$$

Combining the above two equations, we obtain the identity for problem (1.3).

Proof of Theorem 1.1 We only prove the conclusion for problem (1.3), the proof for problem (1.4) is the same. By Lemma 2.1,

$$
\int_{\mathbb{R}_{+}^{N}}|u|^{p+1} d x<\infty, \quad \int_{\partial \mathbb{R}_{+}^{N}}|u|^{q+1} d x^{\prime}<\infty \quad \text { and } \quad \int_{\mathbb{R}_{+}^{N}}|\nabla u|^{2} d x<\infty .
$$

Hence, there exists a sequence $R_{n} \rightarrow \infty$ such that

$$
\begin{aligned}
& \frac{R_{n}}{2} \int_{\partial B_{R_{n}}^{+}}|\nabla u|^{2} d S \rightarrow 0, \\
& R_{n} \int_{\partial B_{R_{n}}^{+}}\left|\frac{\partial u}{\partial v}\right|^{2} d S \rightarrow 0, \\
& \frac{R_{n}}{p+1} \int_{\partial B_{R_{n}}^{+}}|u|^{p+1} d S \rightarrow 0,
\end{aligned}
$$

and

$$
\frac{R_{n}}{q+1} \int_{\partial \Gamma_{1}^{R_{n}}}|u|^{q+1} d S \rightarrow 0
$$

as $n \rightarrow \infty$. So we deduce from the Pohozaev identity that

$$
\frac{N-2}{2} \int_{\mathbb{R}_{+}^{N}}|\nabla u|^{2} d x=\frac{N}{p+1} \int_{\mathbb{R}_{+}^{N}}|u|^{p+1} d x+\frac{N-1}{q+1} \int_{\partial \mathbb{R}_{+}^{N}}|u|^{q+1} d x^{\prime}
$$

On the other hand, if we multiply (1.3) by $u$ and integrate by parts, then we obtain

$$
\int_{\mathbb{R}_{+}^{N}}|\nabla u|^{2} d x=\int_{\mathbb{R}_{+}^{N}}|u|^{p+1} d x+\int_{\Gamma_{1}}|u|^{q+1} d x^{\prime}
$$


By the assumptions on the exponent $p, q$, we deduce from (2.13) and (2.14) that

$$
\int_{\mathbb{R}_{+}^{N}}|\nabla u|^{2} d x=0
$$

or

$$
\int_{\mathbb{R}_{+}^{N}}|u|^{p+1} d x=0
$$

hence we have $u \equiv 0$.

\section{Competing interests}

The authors declare that they have no competing interests.

\section{Authors' contributions}

All authors contributed equally to the writing of this paper. All authors read and approved the final manuscript.

\section{Acknowledgements}

The work is supported by National Natural Science Foundation of China (No:11271170) and GAN PO 555 program of Government of Jiangxi Province.

Received: 18 July 2015 Accepted: 15 October 2015 Published online: 05 November 2015

\section{References}

1. Gidas, B, Spruck, J: A priori bounds of positive solutions of nonlinear elliptic equations. Commun. Partial Differ. Equ. 6, 883-901 (1981)

2. Gidas, B, Spruck, J: Global and local behavior of positive solutions of nonlinear elliptic equations. Commun. Pure Appl. Math. 34, 525-598 (1981)

3. Bahri, A, Lions, PL: Solutions of superlinear elliptic equations and their Morse indices. Commun. Pure Appl. Math. 45 1205-1215 (1992)

4. Ramos, M, Terracini, S, Troestler, C: Superlinear indefinite elliptic problems and Pohozaev type identities. J. Funct. Anal. 159, 596-628 (1998)

5. Harrabi, A, Ahmedou, M, Rebhi, S, Selmi, A: A priori estimates for superlinear and subcritical elliptic equations: the Neumann boundary condition case. Manuscr. Math. 137, 525-544 (2012)

6. Harrabi, A, Rebhi, S, Selmi, S: Solutions of superlinear equations and their Morse indices I. Duke Math. J. 94, 141-157 (1998)

7. Harrabi, A, Rebhi, S, Selmi, S: Solutions of superlinear equations and their Morse indices II. Duke Math. J. 94, 159-179 (1998)

8. Yu, X: Solutions of mixed boundary problems and their Morse indices. Nonlinear Anal. TMA 96, 146-153 (2014)

9. $\mathrm{Yu}, \mathrm{X}$ : Liouville theorem for elliptic equations with nonlinear boundary value conditions and finite Morse indices. J. Math. Anal. Appl. 421, 436-443 (2015)

10. Yu, X: Solutions of fractional Laplacian equations and their Morse indices. J. Differ. Equ. 260(1), 860-871 (2016)

11. Yu, X: Liouville Type Theorems for Two Mixed Boundary Value Problems with General Nonlinearities. arXiv:1410.5157

\section{Submit your manuscript to a SpringerOpen ${ }^{\circ}$ journal and benefit from:}

- Convenient online submission

Rigorous peer review

- Immediate publication on acceptance

- Open access: articles freely available online

- High visibility within the field

- Retaining the copyright to your article 